\title{
A common missense single nucleotide polymorphism in the E-selectin gene is significantly associated with essential hypertension in the Han population but only weakly associated in the Uygur population
}

\author{
Zhong Wang ${ }^{1,3}$, Yujun $\mathrm{Xu}^{2,3}$, Shaoze Chen ${ }^{1}$, Li Wang ${ }^{1}$, Hu Ding ${ }^{2}$, Guilin Lu${ }^{1}$, Daowen Wang ${ }^{2}$, Zhihong Zhai ${ }^{1}$, \\ Juncang Duan ${ }^{1}$ and Wangqiang Zhang ${ }^{1}$
}

Experimental and clinical observations suggest that E-selectin could have an important role in essential hypertension (EH), but the relationship between common E-selectin variants and EH has not been extensively studied in the Chinese population. In this study, we explored the association between two common variants in the E-selectin gene (rs5361A/C and rs5355C/T) and EH in the Uygur, Kazakh and Han populations in the Xinjiang area. A case-control study was conducted to explore the association between these two single-nucleotide polymorphisms and EH in a large sample size, including $941 \mathrm{EH}$ subjects (309 Uygur, 264 Kazakh and 368 Han individuals) and 924 control subjects (300 Uygur, 275 Kazakh and 349 Han individuals). Univariate analysis showed that the rs5361 A/C polymorphism was significantly associated with $\mathrm{EH}$ in the Uygur $(P=0.002)$ and Han $\left(P=3.6 \times 10^{-7}\right)$ populations. The CC genotype of this SNP was present only in patients with EH in all of the three nationalities studied. Han individuals who carry the CC genotype of rs5361 were more susceptible to $\mathrm{EH}$, according to the dominant models $\left(P=1.13 \times 10^{-4}\right.$, odds ratio $=3.812,95 \%$ confidence interval: $\left.1.685-7.792\right)$, but there was no association of genotype with EH for the other ethnicities. No significant difference in rs5355 C/T polymorphism rate was found between the $\mathrm{EH}$ and control groups. Our results indicate that the common variant rs5361 is strongly associated with EH in Han individuals and weakly associated in Uygur individuals. The CC genotype of rs5361 might be an independent risk factor for EH among Uygur, Kazakh and Han individuals in the Xinjiang area.

Hypertension Research (2012) 35, 413-417; doi:10.1038/hr.2011.204; published online 8 December 2011

Keywords: E-selectin; essential hypertension; single nucleotide polymorphisms

\section{INTRODUCTION}

Essential hypertension $(\mathrm{EH})$ is one of the largest public health issues threatening human health worldwide. The morbidity and mortality from EH-induced coronary heart disease, stroke and renal events account for an increasingly large portion of all morbidity and mortality each year. It has been estimated that by 2020, coronary heart disease and stroke will be the first and fourth most common causes of morbidity worldwide, respectively. Human E-selectin, a proinflammatory cytokine also known as the leukocyte differentiation antigen CD62E, is closely associated with EH. The gene encoding E-selectin is located on the long arm of chromosome 1, and it contains 14 exons and 13 introns spanning $\sim 13$ kilobases of the human genome. Recently, several E-selectin gene polymorphisms were suggested to be associated with $\mathrm{EH}^{1-2}$ Marteau et al. ${ }^{3}$ found that the Leu554Phe polymorphism (rs5355) in E-selectin is associated with elevated blood pressure (BP) in overweight people. An age-specific effect of this polymorphism on BP levels was also suggested by Sass et al. ${ }^{4}$ Wenzel et al. ${ }^{5}$ revealed that two mutations, Ser128Arg and Leu554Phe, could influence E-selectin function in vitro and may be considered as risk factors in the complex pathogenesis of atherosclerosis. It has also been suggested that the Ser128Arg polymorphism can functionally alter leukocyte-endothelial interactions, and may have other biochemical and biological consequences that are relevant to the pathogenesis of myocardial infarction. ${ }^{6}$ On the basis of these results, we hypothesized that rs5361 may also have a role in the pathogenesis of hypertension. Therefore, we genotyped two single-nucleotide polymorphisms in Eselectin, rs5361 A/C and rs5355 C/T, using TaqMan allelic discrimination assays (Shanghai GeneCore BioTechnologies Co., Ltd., Shanghai, China) to explore the association between these polymorphisms and $\mathrm{EH}$ among Uygur, Kazakh and Han individuals in the Xinjiang area.

${ }^{1}$ The Department of Cardiology, The First Affiliated Hospital, Shihezi Medical College, Shihezi University, Xingjiang, PR China and ${ }^{2}$ The Institute of Hypertension and Department of Internal Medicine, Tongii Hospital, Tongii Medical College, Huazhong University of Science and Technology, Wuhan, China

${ }^{3}$ These authors contributed equally to this work.

Correspondence: Dr Z Wang, The Department of Cardiology, First Affiliated Hospital, Shihezi Medical College, Shihezi University, Shihezi, Xinjiang 832008, PR China, E-mail: wangzshz@163.com

Received 26 March 2011; revised 21 August 2011; accepted 25 September 2011; published online 8 December 2011 


\section{METHODS}

\section{Subjects}

A total of 941 individuals, aged 30-76 years at recruitment (309 Uygur, 264 Kazakh and 368 Han), were enrolled in an epidemiological survey focusing on hypertension prevalence that was launched in four geographical regions within the Xinjiang province of China in 2008. The control group, consisting of 924 unrelated individuals (300 Uygur, 275 Kazakh and 349 Han), was initially recruited in the same study and matched to cases by sex, race, geographic location and date of collection (within 3 months). All participants underwent standard medical history and physical evaluations, including an assessment of smoking history, stroke, diabetes, coronary artery disease status and the use of antihypertensive medications within 2 weeks of the epidemiological survey. Plasma glucose, total cholesterol, high-density lipoprotein cholesterol and triglyceride levels were measured using standard methods with an OLYMPUS AU 2700 automatic biochemical analyzer (Olympus CO Ltd., Tokyo, Japan), after the patients had fasted for $12 \mathrm{~h}$ overnight. The low-density lipoprotein cholesterol concentration was estimated by the Friedewald formula. ${ }^{7} \mathrm{EH}$ was defined as a systolic BP $\geqslant 140 \mathrm{~mm} \mathrm{Hg}$ or diastolic BP $\geqslant 90 \mathrm{~mm} \mathrm{Hg}$ in the absence of treatment with antihypertensive drugs. Subjects with $\mathrm{EH}$ who did not receive any antihypertensive therapy in the 2 weeks before the epidemiological survey and developed neither complications nor clinical manifestations were included in this study. Participants were excluded from analyses if they (1) had pre-hypertension or received any antihypertensive medication, (2) had severe hepatic or renal dysfunction, secondary hypertension or severe heart failure or (3) were pregnant, lactating or taking oral contraceptives. The local bioethics committee approved the study protocol, and informed consent was obtained from all participants prior to their participation in the study.

\section{DNA extraction and genotyping}

Fasting venous blood was collected in $5 \mathrm{ml}$ EDTA tubes, and genomic DNA was isolated with a DNA extraction kit (FUJIFILM, Tokyo, Japan) according to the manufacturer's protocol. Genotyping was performed with the TaqMan SNP allelic discrimination assay. TaqMan Universal PCR Master Mix $(2 \times)$ was obtained from Applied Biosystems (Carlsbad, CA, USA). Fluorescent probes and PCR amplification primers for the two alleles were designed and synthesized by Shanghai GeneCore BioTechnologies (Shanghai, China). All fluorescent probes were TaqMan TAMRA probes consisting of a TAMRA modifying group, a $5^{\prime}$-reporter dye and a $3^{\prime}$-non-fluorescent quencher. The probe and primer sequences are listed in Table 1.

\section{PCR amplification system, reaction conditions and genotype analysis}

Amplifications were performed in an ABI $7900 \mathrm{HT}$ thermal cycler (Applied Biosystems) with a PCR temperature profile consisting of incubation at $50^{\circ} \mathrm{C}$ for $2 \mathrm{~min}$, denaturation at $95^{\circ} \mathrm{C}$ for $10 \mathrm{~min}$, and 50 cycles of denaturation at $95^{\circ} \mathrm{C}$ for $15 \mathrm{~s}$ and annealing at $60^{\circ} \mathrm{C}$ for 60 s. Each PCR amplification reaction contained $5 \mu \mathrm{l}$ of $1 \times$ Universal Master Mix (Applied Biosystems) containing $0.2 \mu \mathrm{M}$ each of two probes, $1 \mu \mathrm{M}$ each primer and $1 \mathrm{ng} \mu \mathrm{l}^{-1}$ genomic DNA. The genotypes of the two alleles were differentiated with SDS 2.1 software (Applied Biosystems) (Supplementary Figure 1), and 10\% of all genotyping reactions were repeated in independent PCRs to check for consistency and to ensure intraplate and interplate reproducibility. No discrepancies were detected between the repeated samples. In addition, all paired case and control DNA samples were run in the same batches.
Table 1 Sequences of probes and primers

\begin{tabular}{ll}
\hline Primer (5'-3') & Probe (5'-3') \\
\hline F 5'-TAATAGTCCTCCTCATCATGCTTTG-3' & 5'-FAM-ATACATCCTGCAGTGGCCACGGT-TAMRA-3' \\
R 5'-CAAGTGTAATTATTGATGGTCTCTACACA-3' & 5'-HEX-TACATCCTGCCGTGGCCACG-TAMRA-3' \\
F 5'-CCACTGAGTCCAACATTCCCTT-3' & 5'-FAM-ATTAGCACCATTTCTCCTCTGGCTTCG-TAMRA-3' \\
R 5'-TCATAAACTTCCTCACCTTTCCGTA-3' & 5'-HEX-ATTAGCACCATTTTTCCTCTGGCTTCG-TAMRA-3' \\
\hline
\end{tabular}

Abbreviations: F, forward primer; FAM and HEX, fluorescence dyes; R, reverse primer; TAMRA, TAMRA-quenched probe.

\section{Statistical analysis}

Statistical analysis was conducted with SPSS 13.0 (SPSS Inc., Chicago, IL, USA). Continuous variable data that fit a normal distribution were expressed as the mean \pm s.d. $\chi^{2}$-tests were employed to test whether the genotypes were in Hardy-Weinberg equilibrium, and were also used to compare the genotype and allele frequencies between the $\mathrm{EH}$ and control groups. All analyses were performed separately by race. Unconditional logistic regression was performed to estimate the odds ratios (OR) and 95\% confidence intervals (CI). Covariates included gender, age, body mass index, smoking status, diabetes, stroke and coronary artery disease. The study had an $80 \%$ power to detect an association with an OR $>2.80$ (assuming a risk effect) for minor alleles at a $1.4 \%$ frequency, assuming a two-sided significance level at 0.05 and a dominant gene model. The QUANTO program (developed by Jim Gauderman and John Morrison, http://hydra.usc.edu/gxe) was used to construct the model. ${ }^{8}$

\section{RESULTS}

\section{Subject characteristics}

Demographic details for the case and control groups are given in Table 2. There were no significant differences in age or gender between case and control groups. In all three ethnicities, systolic BP and diastolic BP were higher among cases than among controls. The prevalence of coronary artery disease (CAD) and hyperlipidemia was higher in hypertensive subjects than in controls in the Uygur population. The hypertensive group contained a higher proportion of smokers in the Han population but not in the other two populations. Significantly higher serum total cholesterol, triglyceride (TG) and lowdensity lipoprotein levels were identified in the case group for all three ethnicities. However, higher concentrations of fasting glucose were observed only in Han hypertensive subjects, whereas higher highdensity lipoprotein levels were seen in Kazakh and Uygur hypertensive subjects. The genotype distributions of the two single-nucleotide polymorphisms (rs5361 and rs5355) were in Hardy-Weinberg equilibrium in all control groups, and the minor allele frequencies in the controls were very similar to those published for the Han population in the NCBI database (Table 3), indicating that the selected group was representative of the population as a whole.

\section{Association analysis}

Association statistics results are listed in Table 4. Univariate analysis showed that rs5361 A/C polymorphism was significantly associated with $\mathrm{EH}$ in the Uygur $(P=0.002)$ and Han $\left(P=3.6 \times 10^{-7}\right)$ populations. No significant correlation was found in the Kazakh population $(P=0.172)$. The CC genotype of rs5361 was found only in the $\mathrm{EH}$ group in all three nationalities, with frequencies of $4.6 \%, 0.4 \%$ and $3.5 \%$ for the Uygur, Kazakh and Han populations, respectively. Multivariate unconditional logistic regression analysis showed that rs5361 polymorphism was significantly associated with $\mathrm{EH}$, independent of traditional cardiovascular risk factors, in a dominant model in the Han cohort $\left(P=1.13 \times 10^{-4}, \mathrm{OR}=3.81,95 \% \mathrm{CI}: 1.685-7.792\right)$. No effect was identified in the other two ethnicities. 
Table 2 Baseline characteristics of three ethnic samples

\begin{tabular}{|c|c|c|c|c|c|c|}
\hline \multirow[b]{2}{*}{ Characteristics } & \multicolumn{2}{|c|}{ Kazak } & \multicolumn{2}{|c|}{ Uygur } & \multicolumn{2}{|c|}{ Han } \\
\hline & Controls $(n=275)$ & Hypertension $(n=264)$ & Controls $(n=300)$ & Hypertension $(n=309)$ & Controls $(n=349)$ & Hypertension $(n=368)$ \\
\hline Men (\%) & 41.1 & 45.3 & 51.1 & 53.6 & 49.4 & 52.7 \\
\hline BMI $\left(\mathrm{kg} \mathrm{m}^{-2}\right)$ & $24.2 \pm 3.8$ & $26.6 \pm 4.3^{*}$ & $27.0 \pm 4.5$ & $27.6 \pm 3.4$ & $23.56 \pm 3.18$ & $25.48 \pm 2.83^{*}$ \\
\hline $\mathrm{SBP}(\mathrm{mm} \mathrm{Hg})$ & $118 \pm 11$ & $150 \pm 22^{*}$ & $122 \pm 8$ & $156 \pm 13^{*}$ & $116.7 \pm 10.3$ & $147.0 \pm 13.7^{*}$ \\
\hline CAD (\%) & 12.9 & 17 & 0.0 & $34.4^{*}$ & 2.3 & 5.4 \\
\hline Hyperlipidemia (\%) & 5.2 & $12.5^{*}$ & 30.7 & $43.0^{*}$ & 2.6 & 2.5 \\
\hline DM (\%) & 2.6 & 2.7 & 1.4 & 1.6 & 3.1 & 3.3 \\
\hline Smokers (\%) & 8.4 & 6.4 & 21.4 & 21.0 & 24.6 & $34.7^{*}$ \\
\hline Total cholesterol (mg dl $\left.{ }^{-1}\right)$ & $171.15 \pm 39.00$ & $193.82 \pm 40.54^{*}$ & $164.09 \pm 29.73$ & $191.51 \pm 28.57^{*}$ & $166.41 \pm 32.43$ & $177.99 \pm 35.14^{*}$ \\
\hline Triglycerides (mg dl-1) & $101.77 \pm 85.84$ & $123.01 \pm 90.27 *$ & $115.93 \pm 110.62$ & $162.83 \pm 110.62^{*}$ & $109.73 \pm 146.02$ & $160.18 \pm 149.56^{*}$ \\
\hline
\end{tabular}

Abbreviations: BMI, body mass index; CAD, coronary artery disease; DBP diastolic blood pressure; DM, diabetes milletes; HDL, high-density lipoprotein; LDL, low-density lipoprotein; SBP, systolic blood pressure; yrs, years.

Continuous variables are given as mean \pm s.d. ${ }^{*} P<0.05$ vs. control, using $t$ test for continuous variables and $\chi^{2}$-test for categorical variables.

Table 3 Genetic diversity in different ethnic population on rs5361 and rs5355

\begin{tabular}{lccc}
\hline & \multicolumn{3}{c}{ MAF from NCBI } \\
\cline { 2 - 4 } SNP & CEU & CHB & JPT \\
\hline rs5361 & 0.092 & 0.011 & 0.023 \\
rs5355 & 0.025 & 0.011 & 0.068
\end{tabular}

Abbreviations: CEU, population of western European ancestry; CHB, Han Chinese in Beijing; JPT, Japanese in Tokyo, Japan; MAF, minor allele frequency; SNP, single nucleotide polymorphism.

For rs5355 C/T, no significant correlation was found between gene polymorphism and $\mathrm{EH}$ for any of the three nationalities $(P=0.077$ for Uygur, $P=0.27$ for Kazakh and $P=0.635$ for Han) in the univariate analyses. Additionally, multivariate logistic regression using a dominant model did not reveal any significant relationship between rs5355 polymorphism and $\mathrm{EH}$ in these three ethnicities.

\section{DISCUSSION}

In 2001, Bellelli et al. ${ }^{9}$ reported that the serum level of high-sensitive C-reactive protein was an independent risk factor for EH. It has generally been thought that inflammatory injury of the vascular endothelial tissue has an important role in the pathology of $\mathrm{EH}$, and E-selectin-mediated leukocyte adhesion and aggregation is thought to be a prerequisite for inflammatory injury of the vascular endothelia. Although the inflammatory response in the vascular endothelia can be mediated by many kinds of adhesion molecules, the role of E-selectin cannot be performed by other adhesion molecules, and E-selectin is therefore essential for this response. ${ }^{10}$

E-selectin was first identified in 1985 as a transmembrane glycoprotein that is synthesized by vascular endothelial cells after their activation by inflammatory factors and then released from the cell surface as a proinflammatory cytokine. E-selectin was later found to mediate leukocyte adhesion to endothelial cells and their subsequent penetration of the vascular wall. ${ }^{11}$ It is now clear that inflammatory factors affect BP by inducing the expression of adhesion molecules, mainly E-selectin, in endothelial tissue. Generally, E-selectin induces the adhesion and aggregation of leukocytes, which subsequently migrate into the vascular wall or extravascular space, thus injuring vascular endothelial cells, impairing endothelium-dependent vascular relaxation and finally leading to hypertension. Conversely, hypertension also contributes to inflammatory responses through hemodynamic mechanisms. ${ }^{12}$ Thus, hypertension escalates through a positive feedback loop in which inflammation and hypertension promote one another. ${ }^{13}$ Furthermore, hypertension induced by E-selectin might also be related to microcirculation abnormalities; changes in microcirculation structure and function might result from and subsequently contribute to a high BP. ${ }^{14}$ Hypertension is accompanied by a series of morphological changes, such as increased wall-tolumen area ratios in small resistance arteries and decreased capillary network densities in the vascular bed, ${ }^{15}$ which might maintain and enhance BP by increasing peripheral resistance. E-selectin has also been found to impair microcirculation integrity. By promoting the adhesion of leukocytes to endothelial cells and leukocyte-endothelia interactions, enhancing the production of cytokines as well as the products of cytokine-catalyzed reactions in migrated and aggregated leukocytes, ${ }^{16}$ E-selectin leads to changes in microvessels, such as the reconstruction of smooth muscle cells, finally affecting BP. Meanwhile, E-selectin-mediated injury of the vascular endothelia induces several complications of hypertension, such as atherosclerotic vascular disease. This is consistent with the established relationship between E-selectin gene polymorphism or serum soluble E-selectin level and atherosclerotic vascular disease, such as coronary heart disease, ischemic stroke and kidney injury. ${ }^{6,17,18}$ Therefore, E-selectin is intricately involved in the etiology of hypertension.

Chen HL et al. ${ }^{19}$ found that individuals with a C allele at rs5361 have a relatively higher risk of hypertension, higher diastolic BP and a higher mean arterial pressure. Wang et al. ${ }^{20}$ also suggested that the $\mathrm{C}$ allele at this locus was more frequent among hypertensive individuals, consistent with the results of our study. Meanwhile, the rs5355 C/T polymorphism in the E-selectin gene showed age-specific effects on BP in another study. ${ }^{2}$ However, these conclusions were not fully 
Table 4 Association between rs5361 (A/C), rs5355 (C/T) variants and EH

Adjusted ORs (95\% Cl)

\begin{tabular}{|c|c|c|c|c|c|c|c|c|c|c|}
\hline SNP & Ethnicity & Population & N & MAF & $\mathrm{P}_{\text {allelic }}$ & $M M \mathrm{n},(\%)$ & $M m \mathrm{n},(\%)$ & $m m \mathrm{n},(\%)$ & $\mathrm{P}_{\text {domiant }}$ & $M m+m m$ vs. $M M$ \\
\hline \multirow[t]{6}{*}{ rs5361 (A/C) } & Han & Control & 349 & 0.014 & $3.6 \times 10^{-7}$ & $339(97.1)$ & $10(2.8)$ & 0 & $1.13 \times 10^{-4}$ & 1.0 (ref) \\
\hline & & $\mathrm{EH}$ & 366 & 0.068 & & 329 (89.9) & $24(6.6)$ & $13(3.5)$ & & $3.812(1.685-7.792)$ \\
\hline & Uygur & Control & 299 & 0.038 & 0.002 & $276(92.3)$ & $23(7.7)$ & 0 & 0.102 & 1.0 (ref) \\
\hline & & $\mathrm{EH}$ & 309 & 0.081 & & $273(88.3)$ & $22(7.1)$ & $14(4.6)$ & & $1.582(0.914-2.741)$ \\
\hline & Kazak & Control & 275 & 0.036 & 0.172 & $255(92.7)$ & $20(7.3)$ & 0 & 0.285 & 1.0 (ref) \\
\hline & & $\mathrm{EH}$ & 264 & 0.053 & & $237(89.8)$ & $26(9.8)$ & $1(0.4)$ & & $1.453(0.793-2.659)$ \\
\hline \multirow{5}{*}{ rs5355 (C/T) } & & $\mathrm{EH}$ & 368 & 0.038 & & $340(92.4)$ & $28(7.6)$ & 0 & & $0.909(0.529-1.561)$ \\
\hline & Uygur & Control & 299 & 0.055 & 0.077 & $266(89.0)$ & $33(12.4)$ & 0 & 0.07 & 1.0 (ref) \\
\hline & & $\mathrm{EH}$ & 307 & 0.034 & & $286(93.1)$ & $21(6.9)$ & 0 & & $0.592(0.334-1.049)$ \\
\hline & Kazak & Control & 272 & 0.06 & 0.27 & $239(87.9)$ & $33(12.1)$ & 0 & 0.196 & 1.0 (ref) \\
\hline & & $\mathrm{EH}$ & 264 & 0.046 & & 241 (91.3) & $22(8.3)$ & $1(0.4)$ & & $0.691(0.394-1.212)$ \\
\hline
\end{tabular}

Abbreviations: $\mathrm{Cl}$, confidence interval; $\mathrm{EH}$, essential hypertension; $\mathrm{M}$, major allele; $\mathrm{m}$, minor allele; MAF, minor allele frequency; OR, odds ratio; $P_{\text {allele, }}$ value of allele was determined by a two-sided $\chi^{2}$ test; $P_{\text {dominant }}$, value and adjusted odds ratio ( $95 \%$ confidence interval) were computed with multivariate logistic regression analysis by adjusting for gender, age, body mass index, hypertension, diabetes, hyperlipidemia and smoking status; ref, reference; SNP, single nucleotide polymorphism.

confirmed. Our study demonstrated that this polymorphism was not significantly correlated with $\mathrm{EH}$ in the Uygur, Kazakh or Han populations in Xinjiang area, whereas rs5361 A/C was significantly correlated with $\mathrm{EH}$ in the Han population but only mildly in the Uygur population $\left(P=3.6 \times 10^{-7}\right.$ and $P=0.002$, respectively). Subjects carrying the $\mathrm{C}$ allele were more susceptible to $\mathrm{EH}$ under the dominant models $\left(P=1.13 \times 10^{-4}, \mathrm{OR}=3.812,95 \% \mathrm{CI}\right.$ : $\left.1.685-7.792\right)$ in the Han population, but this effect was not observed in the other ethnicities. This difference could be attributed to the genetic heterogeneity among these different populations. Some variants may be poor markers for the trait because of differences in linkage-disequilibrium structure in ethnic backgrounds. As no data on linkage-disequilibrium patterns for Uygur and Kazakh populations are available through the HapMap database, we cannot rule out the possibility that there are differences between these groups and the Han population. Furthermore, the minor allele frequency of these two single-nucleotide polymorphisms differed significantly between the Han population and the other two populations (Table 4). It has been shown that even a small change in the minor allele frequency of an SNP can dramatically reduce a study's power to replicate a main effect for the same SNP. ${ }^{21}$ In addition, our sample sizes of Uygur and Kazakh individuals might be not large enough to reliably detect the effect observed in the Han population. More importantly, the risks in the development of hypertension in these ethnicities may be different because of true etiologic heterogeneity, which may be driven by differences in gene-gene or geneenvironment interactions. This hypothesis is supported by the fact that the EH in Kazakh people in the Xinjiang area is characterized by high morbidity and is significantly affected by environment and diet, ${ }^{20}$ and that, among the elderly, non-Han individuals suffer from a greater prevalence of hypertension and a higher body mass index than Han individuals. ${ }^{22}$

The CC genotype at rs5361 was found only in EH patients, with frequencies of $4.6 \%, 0.4 \%$ and $3.5 \%$ for Uygur, Kazakh and Han populations, respectively. These findings suggest that the CC genotype of E-selectin allele rs5361 A/C might be an independent risk factor for $\mathrm{EH}$ in Uygur, Kazakh and Han populations in the Xinjiang area.

In summary, our study found a strong association between rs5361 polymorphism and $\mathrm{EH}$ in the Han population and a weak association in the Uygur population; our results also indicate that the CC genotype might be an independent risk factor for $\mathrm{EH}$ in Uygur, Kazakh and Han individuals in the Xinjiang area. Further research, specifically large-scale prospective cohort studies and further investigation of the biological mechanism underlying the link between E-selectin and essential hypertension, will be needed to confirm these findings.

\section{CONFLICT OF INTEREST}

The authors declare no conflict of interest.

\section{ACKNOWLEDGEMENTS}

This work was supported by grants from the Medical and Health Care Science \& Technology Specific Projects of Xinjiang Production and Construction Corps (no. 2008GG39).

1 Chang YP, Liu X, Kim JD, Ikeda MA, Layton MR, Weder AB, Cooper RS, Kardia SL, Rao DC, Hunt SC, Luke A, Boerwinkle E, Chakravarti A. Multiple genes for essentialhypertension susceptibility on chromosome 1q. Am J Hum Genet 2007; 80: 253-264.

2 Sass C, Pallaud C, Zannad F, Visvikis S. Association between E-selectin Leu554Phe polymorphism and blood pressure in the Stanislas cohort]. Arch Mal Coeur Vaiss 2001; 94: 855-858.

3 Marteau JB, Sass C, Pfister M, Lambert D, Noyer-Weidner M, Visvikis S. The Leu554Phe polymorphism in the E-selectin gene is associated with blood pressure in overweight people. J Hypertens 2004; 22: 305-311.

4 Sass C, Pallaud C, Zannad F, Visvikis S. Relationship between E-selectin L/F554 polymorphism and blood pressure in the Stanislas cohort. Hum Genet 2000; 107: 58-61.

5 Wenzel K, Stahn R, Speer A, Denner K, Glaser C, Affeldt M, Moobed M, Scheer A, Baumann G, Felix SB. Functional characterization of atherosclerosis-associated Ser128Arg and Leu554Phe E-selectin mutations. Biol Chem 1999; 380: 661-667.

6 Yoshida M, Takano Y, Sasaoka T, Izumi T, Kimura A. E-selectin polymorphism associated with myocardial infarction causes enhanced leukocyte-endothelial interactions under flow conditions. Arterioscler Thromb Vasc Biol 2003; 23: 783-788.

7 Friedewald WT, Levy RI, Fredrickson DS. Estimation of the concentration of low-density lipoprotein cholesterol in plasma, without use of the preparative ultracentrifuge. Clin Chem 1972; 18: 499-502.

8 Knight J. A survey of current software for genetic power calculations. Hum Genomics 2004; 1: 225-227.

9 Bellelli G, Rozzini R, Battista Frisoni G, Trabucchi M. Is C-reactive protein an independent risk factor for essential hypertension? J Hypertens 2001; 19: 2107.

10 Forlow SB, White EJ, Barlow SC, Feldman SH, Lu H, Bagby GJ, Beaudet AL, Bullard DC, Ley K. Severe inflammatory defect and reduced viability in CD18 and E-selectin double-mutant mice. J Clin Invest 2000; 106: 1457-1466. 
11 Bevilacqua MP, Stengelin S, Gimbrone Jr MA, Seed B. Endothelial leukocyte adhesion molecule 1: an inducible receptor for neutrophils related to complement regulatory proteins and lectins. Science 1989; 243: 1160-1165.

12 Blake GJ, Rifai N, Buring JE, Ridker PM. Blood pressure, C-reactive protein, and risk of future cardiovascular events. Circulation 2003; 108: 2993-2999.

13 Tatasciore A, Zimarino M, Renda G, Zurro M, Soccio M, Prontera C, Emdin M, Flacco M, Schillaci G, R DEC. Awake blood pressure variability, inflammatory markers and target organ damage in newly diagnosed hypertension. Hypertens Res 2008; 31: 2137-2146.

14 Levy BI, Ambrosio G, Pries AR, Struijker-Boudier HA. Microcirculation in hypertension: a new target for treatment? Circulation 2001; 104: 735-740.

15 Antonios TF, Singer DR, Markandu ND, Mortimer PS, MacGregor GA. Rarefaction of skin capillaries in borderline essential hypertension suggests an early structural abnormality. Hypertension 1999; 34(4 Part 1): 655-658.

16 Lusis AJ. Atherosclerosis. Nature 2000; 407: 233-241.
17 Dehne MG, Sablotzki A, Muhling J, Hartmann B, Rohrig R, Meister M. Evaluation of SE-Selectin and SICAM-1 as parameters for renal function. Ren Fail 2008; 30: 675-684.

18 Zak I, Sarecka B, Krauze J. Synergistic effects between 561A $>$ C and 98G > T polymorphisms of $\mathrm{E}$-selectin gene and hypercholesterolemia in determining the susceptibility to coronary artery disease. Heart Vessels 2008; 23: 257-263.

19 Chen HL, Hua Q, Liu RK, Yang Z. Effect of E-selectin A561C (S128R) polymorphism on blood pressure. Zhonghua Xin Xue Guan Bing Za Zhi 2005; 33: 603-607.

20 Wang Z, Liu Y, Liu J, Liu K, Lou Y, Wen J, Niu Q, Wen S, Wu Z. E-selectin gene polymorphisms are associated with essential hypertension: a case-control pilot study in a Chinese population. BMC Med Genet 2010; 11: 127.

21 Greene CS, Penrod NM, Williams SM, Moore JH. Failure to replicate a genetic association may provide important clues about genetic architecture. PLOS One 2009; 4: e5639.

22 Lu Z, Zhu Y, Yan Z, Liu X, Yan W, Shi GP. Enhanced hypertension prevalence in non-Han Chinese minorities from Xinjiang Province, China. Hypertens Res 2009; 32: 1097-1103.

Supplementary Information accompanies the paper on Hypertension Research website (http://www.nature.com/hr) 